Service social

\title{
La fréquentation d'un centre de jour pour personnes âgées : les effets sur la relation avec le réseau de support naturel
}

\section{Lorraine Brissette}

Volume 34, numéro 1, 1985

Personnes âgées, milieux de vie et pratiques sociales

URI : https://id.erudit.org/iderudit/706250ar

DOI : https://doi.org/10.7202/706250ar

Aller au sommaire du numéro

Éditeur(s)

École de service social de l'Université Laval

ISSN

1708-1734 (numérique)

Découvrir la revue

Citer cet article

Brissette, L. (1985). La fréquentation d'un centre de jour pour personnes âgées : les effets sur la relation avec le réseau de support naturel. Service social, 34(1), 46-54. https://doi.org/10.7202/706250ar d'utilisation que vous pouvez consulter en ligne. 
BRISSETTE, Lorraine, praticienne sociale au C.S.S. du Centre du Québec à Louiseville.

\section{La fréquentation d'un centre de jour pour personnes âgées : les effets sur la relation avec le réseau de support naturel}

\section{Lorraine Brissette}

Depuis quelques années, les travaux sur les réseaux sociaux se multiplient. Cette nouvelle approche de la réalité sociale nous semble particulièrement appropriée à l'étude des problèmes affectant les personnes âgées. Elle nous ouvre, surtout en tant que praticiens, des pistes de réflexion et d'action fort intéressantes. Les données et les idées présentées dans cet article sont en grande partie inspirées d'une étude exploratoire réalisée dans le cadre d'une thèse de maîtrise en service social portant sur la comparaison des réseaux de support naturel de deux groupes de personnes âgées. ${ }^{1}$ Le premier est composé de personnes âgées fréquentant le centre de jour depuis plus de deux ans et le deuxième, de personnes attendant leur admission.

Cette recherche avait pour objectif de connaitre l'influence d'un centre de jour pour personnes âgées en tant que ressource formelle sur le réseau de support naturel de l'usager et sur sa stratégie de solution de problèmes. Les principaux résultats démontrent que la fréquentation du centre de jour n'a pas entraîné de modifications dans les réseaux de support naturel et qu'ils demeurent la principale et quasi unique ressource qu'utilisent les personnes âgées dans le processus de solution de problèmes. La recension des écrits nous a permis d'identifier deux facteurs qui peuvent expliquer conjointement ces résultats, soit la typologie familiale et le recours aux services rattaché à cette typologie.

Les études du sociologue américain Kerckoff ${ }^{2}$ lui ont permis d'identifier trois types de familles. Dans le premier, la «famille 
étendue ", les attentes des parents quant au support de leurs enfants sont grandes et ceux-ci vivent près d'eux. Cette catégorie reste attachée aux valeurs et aux répartitions traditionnelles des tâches. Elle est peu perméable au changement et on y retrouve de fréquents conflits entre la réalisation de l'individu et les valeurs familiales. Leur chef occupe généralement un emploi d'ouvrier, de col bleu, d'agriculteur ; il possède un faible niveau de scolarité et est peu enclin à la mobilité.

Le deuxième type s'oppose radicalement au premier: c'est la "famille nucléaire». Les parents y nourrissent peu d'attentes de support de la part de leurs enfants et ceux-ci vivent en des endroits plus éloignés. On y retrouve un partage de tâches plus égalitaire. Le changement y est perçu comme bénéfique, la mobilité plus grande et la famille moins nombreuse. Le chef occupe généralement un emploi de col blanc et possède un niveau supérieur d'instruction. II existe peu de conflits entre les valeurs familiales et les efforts des enfants pour s'élever de la couche sociale dont ils sont issus.

Finalement, dans le troisième type, la "famille étendue et modifiée" les parents souhaitent obtenir de l'aide de leurs enfants, mais sans leur proximité. La quasi-totalité des personnes âgées de nos échantillons provenaient de la première catégorie.

Nous pouvons relier cette typologie de Kerckoff à l'étude de McKinlay ${ }^{3}$ portant sur la référence et l'utilisation des services publics par les familles. La principale observation de McKinlay est à l'effet que plus celles-ci ont un réseau de relations serré, moins leurs membres utilisent les services publics. Les caractéristiques des familles qui utilisent peu les services publics se rapprochent donc très fortement de la famille de type étendu de Kerckoff. Il apparaît que celles à réseau fortement interrelié (close knit) tentent d'abord de résoudre leurs problèmes par leurs propres moyens; ils ne réfèrent aux services publics qu'en dernier recours.

Les observations de notre recherche vont dans le sens des conclusions de McKinlay. Le processus de solution de problèmes utilisé par nos deux groupes est le même, qu'il y ait ou non fréquentation du centre de jour. Les personnes-ressources utilisées en cas de problème hypothétique sont, en ordre décroissant, un enfant, un parent et un ami.

L'exploration au niveau des problèmes affectifs nous révèle que les personnes âgées des deux groupes utilisent, en premier lieu, dans une large mesure, leur réseau naturel. La deuxième catégorie de ressources utilisées est mixte, c'est-à-dire à la fois formelle et informelle; les ressources formelles seules n'étant utilisées qu'en dernier lieu. L'expérience des entrevues nous permet même de croire que cette utilisation mixte est conditionnée par l'orientation que lui donne 
la proche famille. $\mathrm{Si}$, par exemple, une personne âgée ressent un malaise et qu'elle en avise un enfant, c'est généralement celui-ci qui prend la décision d'appeler ou non le médecin ou l'ambulance selon son évaluation de la situation. C'est donc souvent le niveau de connaissance, d'information et de jugement de la famille proche qui conditionne l'utilisation des services publics par les personnes âgées. L'utilisation des ressources formelles, telles le centre de jour, même sur une base régulière plusieurs heures par semaine et pendant plus de deux ans, n'affecte pas ce comportement de façon notable. C'est davantage le type de famille et les caractéristiques qui lui sont associées qui affectent le plus l'utilisation des services.

\section{L'intervention de réseaux appliquée aux personnes âgées}

Cette donnée se révèle précieuse pour les intervenants. En effet, nos programmes de santé, de services sociaux, d'éducation et d'information pour personnes âgées sont conçus pour s'adresser à des individus et non à des réseaux. L'intervention individuelle, dans les sciences sociales, s'est développée sur des valeurs que l'on retrouve davantage dans les familles de type nucléaire : autonomie, indépendance, respect des différences et des besoins individuels, changement vu comme positif, etc.

Une très large part de la clientèle âgée de nos services publics est issue du type "famille étendue" et fonctionne davantage sur un mode d'interaction de groupe dans ses prises de décision que sur un mode où toute l'autonomie et la responsabilité de choix sont laissées à l'individu. Quand une personne âgée se présente avec un de ses enfants dans nos services et que celui-ci formule une demande d'hébergement, nous avons peut-être tendance à penser qu'il veut placer sa mère ou son père. L'image couramment répandue des enfants qui ne veulent plus s'occuper de leurs parents n'est pas loin.

Cette démarche est vue comme normale par une famille à réseau fortement interrelié où l'opinion du groupe a souvent plus de poids que les opinions individuelles. Plusieurs intervenants peuvent témoigner que même la personne âgée en désaccord avec la demande de placement va se rallier si le refus ou l'hésitation de l'intervenant à accepter la demande risque de causer des conflits ou des scissions au sein de la famille. Pour la personne âgée, c'est souvent le bonheur de ses enfants et la cohésion de sa famille qui priment. L'intervenant doit en être conscient et éviter de mettre le focus de son intervention sur la personne âgée pour uniquement renforcer son autodétermination ou 
pour jouer un rôle d'avocat. II doit davantage porter son action sur le réseau et jouer, dans la mesure du possible, un rôle de médiateur entre les besoins de tous les membres. Ici, c'est le réseau rapproché qui peut être vu à la fois comme clientèle cible et comme ressource.

La persistance du modèle d'utilisation du réseau pour solutionner les problèmes nous porte plus que jamais à croire que l'intervention auprès des personnes âgées doit délaisser le type traditionnel pour adopter un modèle axé sur le réseau. Ce type d'intervention ne doit pas être vu comme niant l'importance de l'individu ou comme aliénant pour la personne âgée. Au contraire, il nous permet de mieux respecter sa dynamique personnelle, qui est intimement liée à sa famille. Les entrevues familiales, les réunions de cuisine regroupant parfois plus de deux générations devraient être monnaie courante en intervention auprès des personnes âgées. La médiation, la conciliation, la recherche de solutions et de formules originales d'entraide, le bris de modèles figés et nocifs de relations familiales devraient aussi être des objectifs courants d'entrevues de groupe.

Plusieurs intervenants connaissent bien ce phénomène où la responsabilité de support de la personne âgée en perte d'autonomie est déléguée à un ou deux membres de son réseau. C'est généralement une femme, soit habitant à proximité, soit célibataire ou ne travaillant pas à l'extérieur. L'épuisement et l'incapacité de ce réseau à soutenir une personne âgée sont bien souvent dus à cette répartition inégale des charges. Seule une approche de groupe axée sur la responsabilisation des membres peut permettre de négocier un arrangement plus adéquat et permettre un maintien de la personne âgée plus longtemps dans son milieu.

Les structures sont-elles prêtes à faire face à un tel changement de pratique? Les lourdes charges de travail des praticiens, la gestion des listes d'attente sont-elles compatibles avec une approche centrée sur le réseau? La formation du personnel est-elle adéquate en ce sens ? II nous reste à espérer que le transfert des responsabilités de maintien à domicile dans les centres locaux de services communautaires (C.L.S.C.) puisse ouvrir la porte à des changements dans l'approche globale des problèmes, dans la pratique quotidienne et dans les allocations de fonds à ces services.

\section{La fréquentation d'un centre de jour et son effet sur la personne âgée}

Si les conclusions de notre étude soulignent l'importance d'une approche de réseau, elles nous apportent en plus des réflexions aussi 
intéressantes qu'inattendues sur l'effet de la fréquentation d'un centre de jour. Nos enquêtes ont porté sur les centres de jour de Loretteville et sur celui du Foyer Joseph-Denys à Trois-Rivières. Nos observations sont valables pour ces deux centres seulement, notre modèle de recherche nous interdisant une généralisation des résultats; elles peuvent cependant fournir des hypothèses sérieuses de travail pour la recherche et l'intervention.

En comparaison avec notre groupe en attente d'admission, plusieurs indicateurs nous démontrent que les personnes âgées qui fréquentent le centre de jour depuis plusieurs années font montre d'un plus grand dynamisme social. Elles ont, dans l'ensemble, une meilleure perception de leur état de santé et de leur situation financière, même si dans les faits leur situation est comparable.

Le réseau de support naturel des "anciens" s'avère être d'une composition plus variée, incluant davantage d'amis et de voisins. Dans l'ensemble de leurs besoins de support, ils dépendent moins de leurs enfants que les nouveaux admis. De plus, ils amorcent davantage les contacts avec les membres de leur réseau plutôt que d'attendre les contacts venant des autres.

Nous avons surtout observé une différence très intéressante au niveau de l'échange de services avec leur réseau. Tant pour les anciens que pour les nouveaux, les personnes âgées en reçoivent beaucoup plus qu'elles n'en rendent. Cependant, au plan socio-affectif, les anciens rendent nettement plus de services à leur réseau que les nouveaux. L'effet principal de la fréquentation du centre de jour n'est donc pas, par exemple, de modifier le rapport au réseau en terme de fréquence de contact mais de changer la personne âgée elle-même.

Si nous regardons les motifs pour lesquels celles du groupe plus ancien ont fait leur demande d'admission, on remarque le besoin de sortir, de rencontrer des gens, de briser l'isolement, de surmonter une crise. Le dynamisme observé quelques années après leur admission démontre bien que le centre de jour a su répondre à leurs attentes.

Dans les commentaires recueillis lors des entrevues, nous avons entendu des personnes âgées nous confier que le centre de jour a amélioré leur état psychologique et leurs rapports avec les autres, que leur curiosité intellectuelle est tenue en éveil et que leur condition physique est devenue meilleure. Une personne nous racontait avec orgueil qu'elle avait elle aussi une vie privée depuis qu'elle fréquentait le centre de jour, qu'elle avait également des choses nouvelles à raconter à ses enfants lors de leurs visites. On peut donc dire que la fréquentation du centre de jour, pour les personnes âgées rencontrées, a enrichi leurs rapports avec leur réseau. 


\section{Le centre de jour et son apport au maintien à domicile}

Les concepteurs de programmes dans les administrations gouvernementales conçoivent théoriquement les centres de jour comme des équipements offrant des services spécialisés et généraux permettant aux personnes âgées de demeurer dans leur milieu. Ils s'intéressent surtout à des services de type instrumental : alimentation, hygiène, nursing, transport, etc.; mais la réalité de nos observations est très différente.

La personne âgée s'appuie toujours sur sa famille et se réfère peu au centre de jour en cas de problème, et fréquente peu les autres bénéficiaires à l'extérieur. En fait, la fréquentation ne change apparemment pas son mode de vie, mais change la personne âgée ellemême intérieurement. Nous émettons l'hypothèse que ce ne sont pas les services instrumentaux eux-mêmes qui ont cet effet, mais les programmes d'activités sociales et éducatives ainsi que les relations nouées à l'intérieur du centre.

Ces observations nous amènent sur des pistes de réflexion en rapport avec le maintien à domicile. Nous savons tous, qu'au niveau des politiques gouvernementales, c'est la priorité de l'heure. On évalue ces services en termes mesurables : budgets, nombre d'heures de service, nombre d'actes de nursing, nombre de bains, d'entrevues, etc. Mais qu'est-ce qui maintient réellement la personne âgée chez elle? Nous identifierons ici trois facteurs pouvant y contribuer.

En premier lieu, les services à domicile eux-mêmes; mais, si nous considérons seulement ceux-ci, nous risquons de tomber dans une illusion dangereuse. Nous ne disposerons jamais d'assez de ressources pour répondre à une demande qui se crée par elle-même. De plus, est-ce qu'un investissement massif dans ceux-là ne pourrait pas entraîner, dans une certaine mesure, une baisse de l'investissement du réseau de support naturel?

La deuxième composante est le réseau lui-même, dans le support qu'il apporte à la personne âgée et dans sa part dans la prise de décision de demeurer chez elle. Nous avons vu finalement que le dynamisme même de la personne âgée est un élément de poids dans son désir de rester à domicile.

C'est donc dire, en troisième lieu, que celles qui ont une perception positive de leur situation, malgré des difficultés réelles, qui se sentent encore utiles aux autres et dont la curiosité intellectuelle est tenue en éveil, tenteront de maintenir leur situation de bien-être psychologique le plus longtemps possible, même en dépit d'une santé chancelante. 
Nous avons fait la connaissance, au cours de l'enquête, d'un certain nombre de personnes âgées dont nous avons pu suivre par la suite la trajectoire de vie. Ces personnes étaient soit seules, soit atteintes de maladies chroniques étant plus ou moins des handicaps; d'autres vivaient des problèmes familiaux aigus; certaines auraient pu être admises au centre d'accueil pendant la période d'enquête ou dans les années qui ont suivi. Trois ans après la cueillette des données, plusieurs fréquentaient encore le centre de jour; d'autres étaient décédées, tout en restant chez elles jusqu'à la fin et très peu avaient fait leur entrée au centre d'accueil.

Ces observations n'ont pas la valeur de données scientifiques mais peuvent cependant nous forcer à nous interroger sur les effets préventifs des centres de jour si notre intention est d'abord de retarder l'hébergement en centre d'accueil. Plus inquiétante encore est l'attitude des technocrates qui imposent des changements d'orientation majeurs aux centres de jour sans étude approfondie de l'impact des programmes existants.

Les orientations actuelles du Ministère des affaires sociales (M.A.S.) vont des centres de jour plutôt psychosociaux vers des centres de jour plus médicaux et axés sur la réadaptation. Elles privilégient la clientèle inscrite sur les listes d'attente pour l'hébergement, donc beaucoup plus lourde, tentant ainsi d'en abréger la durée de fréquentation. En bref, ce serait peut-être une tentative de résoudre un problème de ressources lourdes par des ressources légères de prévention. Où en sera la prévention quand les centres de jour feront face à une clientèle si handicapée qu'elle requiert déjà l'hébergement? Veut-on développer un service d'attente déguisé?

Cette nouvelle orientation des centres de jour vers la santé physique pose le problème de la place qu'occuperont ces ressources par rapport aux C.L.S.C. Si les services de prévention, d'information et de soins de première ligne sont dispensés dans ces derniers pour toute la population, pourquoi les développer également de façon parallèle, dans les centres de jour? Nous appuyant sur les observations de notre étude, nous pouvons avancer que la vocation préventive en santé mentale et en développement personnel devrait y être maintenue et pouvoir desservir une clientèle plus large. Nous sommes bien conscients que ces observations sont fragmentaires et ne concernent que deux centres. Elles indiquent cependant une piste de recherche très importante : les effets au niveau de la santé mentale.

Nous avons identifié précédemment trois composantes du maintien à domicile auxquelles nous pourrions rattacher trois partenaires dans l'intervention (Graphique 1). Le soutien instrumental (soins, hygiène, 


\section{GRAPHIQUE 1}

\section{Composantes du maintien à domicile}

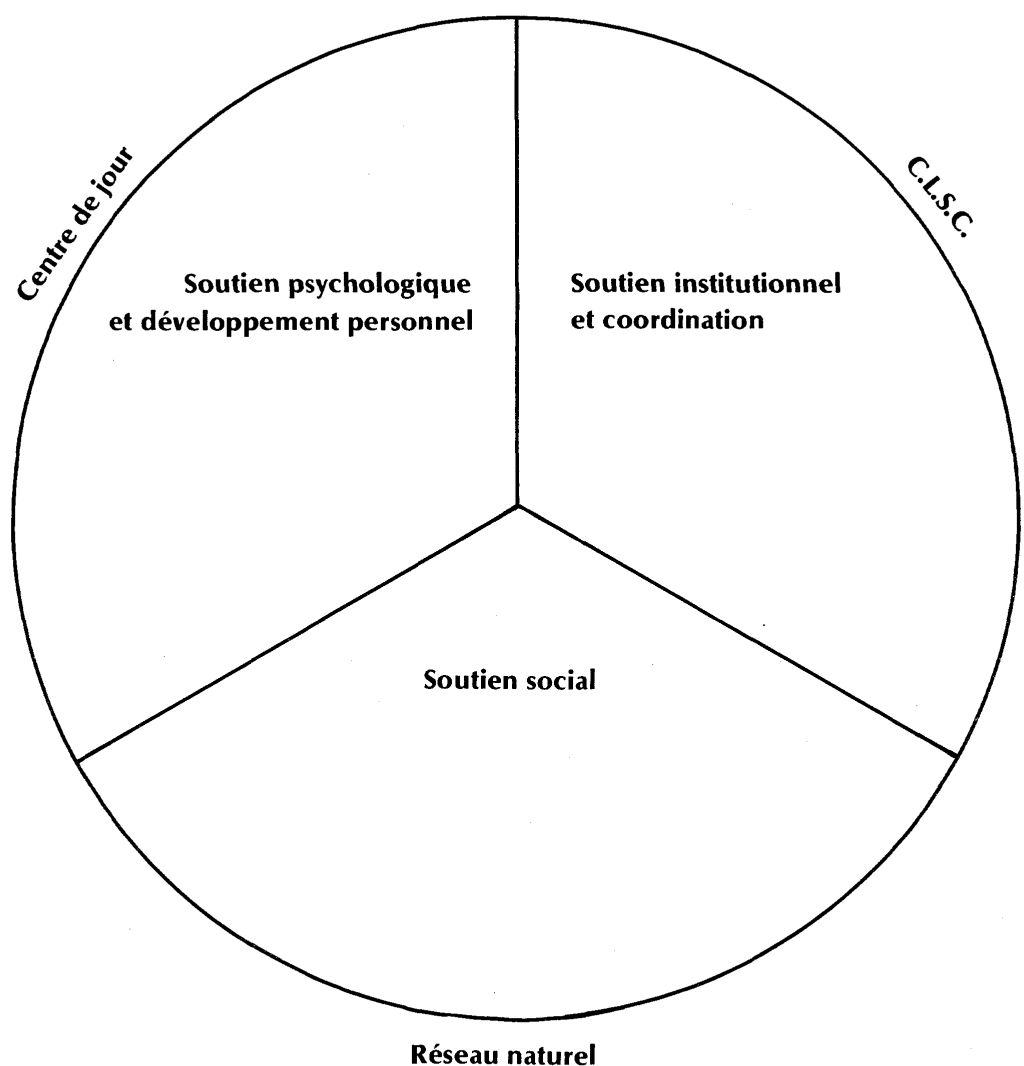

entretien, transport) peut être en partie offert par le C.L.S.C. et par le réseau de support naturel. Les praticiens sociaux rattachés aux C.L.S.C. sont en position pour assurer une coordination efficace des ressources formelles et informelles. Le soutien psychosocial, surtout précieux en période de crise, peut être fourni par le réseau naturel et par le centre de jour qui a déjà fait ses preuves en cette matière. Finalement, le support social est principalement apporté par le réseau naturel, et partiellement par le centre de jour pour ceux qui le fréquentent. 
La proportion des efforts de soutien va évidemment varier en fonction des problématiques et des capacités de la personne âgée et de son réseau. Les efforts des centres de jour et des C.L.S.C. devront être concertés et davantage tenir compte de la réalité des réseaux. Mais, pour réaliser cette concertation, il importe de bien définir les champs d'action propres à chacun.

Cette définition des champs d'intérêt, en ce qui concerne les centres de jour, reste encore à faire. Elle doit dépasser une énumération de services, et avoir des objectifs plus spécifiques que le seul maintien à domicile. Elle doit également identifier les clientèles et les problématiques pour lesquelles ces ressources ont apporté une aide réelle. En fait, le bilan de l'expérience des dix dernières années en cette matière reste encore à faire au Québec. Pourrons-nous enfin bénéficier d'une politique globale du troisième âge définissant et identifiant clairement la place et la complémentarité des divers services, tout en tenant compte de la place et de l'apport des ressources du milieu naturel?

\section{Références bibliographiques}

1 BRISSETTE, L. et V. MORIN, L'influence de la fréquentation des centres de jour sur les réseaux de support naturel des personnes âgées et leurs stratégies de solution de problèmes, Québec, Université Laval, École de service social, 1984.

2 KerCKoff, A.C., "Family pattern and morale in retirement", dans: I.H. SIMPSON et J.C. McKinney (éds.), Social Aspects of Aging, Durham (N.C.), Duke University Press, 1966: 173-194.

3 MCKINLAY, J.B., "Social networks, lay consultation and help-seeking behavior", Social Forces, vol. 52, $\mathrm{n}^{\circ}$ 3, 1973: 275-291. 